\title{
A novel method for evaluating microglial activation using ionized calcium-binding adaptor protein-1 staining: cell body to cell size ratio
}

\author{
Iris Bertha Hovens ${ }^{1}$, Csaba Nyakas ${ }^{1,2}$, Regien Geertruida Schoemaker ${ }^{1,3}$ \\ ${ }^{1}$ Department of Molecular Neurobiology, University of Groningen, 9747 AG, Groningen, The Netherlands. \\ ${ }^{2}$ Department of Brain Physiology Research Unit, Semmelweis University, H-1085, Budapest, Hungary. \\ ${ }^{3}$ Department of Cardiology, University of Groningen, 9747 AG, Groningen, The Netherlands.
}

\section{A B S T R A C T}

\begin{abstract}
Aim: The aim was to validate a newly developed methodology of semi-automatic image analysis to analyze microglial morphology as marker for microglial activation in ionized calcium-binding adaptor protein-1 (IBA-1) stained brain sections. Methods: The novel method was compared to currently used analysis methods, visual characterization of activation stage and optical density measurement, in brain sections of young and aged rats that had undergone surgery or remained naïve. Results: The cell body to cell size ratio of microglia was strongly correlated to the visual characterization activation stage. In addition, we observed specific surgery and age-related changes in cell body size, size of the dendritic processes and cell body to cell size ratio. Conclusion: The novel analysis method provides a sensitive marker for microglial activation in the rat brain, which is quick and easy to perform and provides additional information about microglial morphology.
\end{abstract}

Key words: Image analysis, immunohistochemistry, ionized calcium-binding adaptor protein-1, microglia, neuroinflammation

\section{INTRODUCTION}

Microglia, the primary immune cells in the central nervous system, are highly plastic cells. ${ }^{[1]}$ Under resting conditions, microglia have a ramified morphology, characterized by small cell bodies and numerous long branching processes. ${ }^{[2]}$ The ramified microglia continuously scan the environment for danger signals associated with pathogens or injury. ${ }^{[2,3]}$ When a danger signal is detected microglia undergo a rapid change in morphology and function, a process that has been termed activation. ${ }^{[4]}$ Microglial activation is classically described as a graded process, in which the processes retract and thicken, cell body size increases and the cell starts excreting cytokines and radical species..$^{[1,4,5]}$ Eventually, the microglia may become amoeboid cells capable of phagocytosis. ${ }^{[5]}$ More recently, it has become apparent that depending on the signal detected and local environment microglia can undergo various changes in morphology and function. ${ }^{[2,6]}$ Although microglial

\begin{tabular}{|l|l|}
\hline \multicolumn{2}{|c|}{ Access this article online } \\
\hline Quick Response Code: & \\
\hline & Website: \\
\hline & Www.nnjournal.net \\
& \\
\hline
\end{tabular}

activation provides a defense against injury and infection, chronic or excessive activation is considered to be detrimental and has been implicated in many neurodegenerative and psychiatric disorders. ${ }^{[2,5,7]}$

Ionized calcium-binding adaptor protein-1 (IBA-1) is a $17-\mathrm{kDa}$ actin-binding protein that is specifically and constitutively expressed in all microglia. ${ }^{[8,9]}$ It is widely employed as an immunohistochemical marker for both ramified and activated microglia. ${ }^{[9,10]}$ IBA-1 is shown to have a function in the actin-crosslinking involved in membrane ruffling of microglia. ${ }^{[11]}$ Since membrane ruffling is essential for the morphological changes from quiescent ramified microglia to activated amoeboid microglia, microglial activation is associated with increased IBA-1 expression. ${ }^{[8,11]}$ Densitometry can, therefore, be utilized for measuring microglial activation, especially when microglia is strongly activated and/or microglia number is substantially increased. However, morphological changes of microglia can occur without significantly affecting the optical density (OD) in IBA-1 stained brain areas and densitometry does not provide any specific information on the nature of the morphological changes.

Alternatively, the stadia of microglial activation described by Kreutzberg ${ }^{[4]}$ are often used visually to determine the activation stage of all individual 
microglia in an area of interest, and presenting activation as the percentage of microglia that score above a certain level. This technique provides a more specific and sensitive measurement for microglia activation than densitometry, but is very labor intensive and rather depends on a subjective interpretation of the activation stage. Moreover, this analysis method is solely based on the classical view on microglial activation and as such does not take alternatively morphological changes, such as hyperramification into account. ${ }^{[12,13]}$

In the past years several other methods have been developed that involve morphological characteristics, such as morphological complexity, ${ }^{[14]}$ the extent of ramification $^{[13,15,16]}$ or the length of the dendritic processes. ${ }^{[13,15,16]}$ Although these methods allow a more detailed and accurate analysis of microglial morphology, they still tend to be time consuming and depend on specialized equipment (e.g. a confocal microscope) and software.

Considering the disadvantages of the aforementioned methods, we were in search of a method to determine changes in microglial morphology that is quick and easy to perform, reproducible, and reliable as a microglial activation marker. In this paper, we describe a newly developed semi-automatic image analysis method for IBA-1 stained brain sections of rat. We compare this novel method to the most commonly used methods today, namely densitometry and visual characterization of the activation stage, using IBA-stained sections of rat brains from a previously published experiment. ${ }^{[17]}$

\section{METHODS}

\section{Animals and interventions}

To investigate whether our method could be applied to rat brain sections with a range of activation stadia we reanalyzed IBA-1 stained brain sections from a previously performed experiment in young and aged rats. ${ }^{[17]}$ Male Wistar rats (HsdCpb: WU) of 3 months (young, $n=10$ ) and 18-20 months (old, $n=10$ ) were obtained from a colony of the Semmelweis University (Budapest, Hungary). In each age group, 5 rats were subjected to abdominal surgery under anesthesia ( $3 \%$ sevoflurane in $\mathrm{O}_{2}$ at $0.7 \mathrm{~L} / \mathrm{min}$ ) and buprenorphine analgesia $(0.003 \mathrm{mg} / \mathrm{kg}$ s.c. $)$ and the other 5 remained naïve as control, yielding 4 experimental groups (young control, young surgery, old control and old surgery). Between week 1 and 6 following surgery, the rats underwent several behavioral tests. ${ }^{[17]}$ Animals were sacrificed 6 weeks after the surgical intervention. All experiments were approved by the local animal experiment and welfare committee (Dier Experimenten Commissie, Groningen, The Netherlands).

\section{Immunohistochemistry}

Transcardial perfusion sacrificed animals with saline containing $0.1 \%$ ethylene diamine tetraacetic acid, under pentobarbital anesthesia $(6 \%, 2 \mathrm{~mL} / \mathrm{kg})$. Half of each brain was immersion fixed in $4 \%$ paraformaldehyde for 4 days followed by cryoprotection with $30 \%$ sucrose in $0.01 \mathrm{~mol} / \mathrm{L}$ phosphate-buffered saline (PBS). Free floating sections of $30 \mu \mathrm{m}$ containing the prefrontal cortex (PFC) and the dorsal hippocampus were pretreated with $0.3 \%$ $\mathrm{H}_{2} \mathrm{O}_{2}$ for 20 min. Sections were incubated for 3 days with 1:2500 rabbit-anti IBA-1 (Wako, Neuss, Germany) in $2 \%$ bovine serum albumin, $0.1 \%$ triton $\mathrm{X}-100$ at $4^{\circ} \mathrm{C}$, followed by a $1 \mathrm{~h}$ incubation with 1:500 goat-anti rabbit secondary antibody (Jackson, Wet Grove, USA) at room temperature. The sections were then incubated for $2 \mathrm{~h}$ with avidin-biotin peroxidase complex (Vectastain ABC kit, Vector, Burlingame, USA) at room temperature. Labeling was visualized by using a $0.075 \mathrm{mg} / \mathrm{mL}$ diaminobenzidine (DAB) solution activated with $0.1 \%$ $\mathrm{H}_{2} \mathrm{O}_{2}$. All dilutions were made in $0.01 \mathrm{~mol} / \mathrm{L}$ PBS. All sections were thoroughly rinsed 4 times with 0.01 $\mathrm{mol} / \mathrm{L}$ PBS between staining steps. Sections were mounted onto glass slides in a $1 \%$ gelatin solution and dehydrated through gradients of ethanol and xylol solutions.

\section{Analysis of microglial activation}

Three immunohistochemical labeled sections per area for each rat were analyzed by an operator blinded to the treatment. Average microglial activation was determined in the apical dendritic field (stratum radiatum) of the dentate gyrus inner blade (DGib) and the cornu ammonis 1 (CA1) hippocampal region and in layer III of the PFC (Zilles's Cg1).

\section{Densitometry}

The OD of the IBA-1 stained sections was measured using quantitative imaging software (Leica QWin, Leica Microsystems, Rijswijk, The Netherlands), at $\times 100$ magnification. The OD was corrected according to the background staining measured in the corpus callosum of each individual section. The resulting average OD in each brain area was taken as a measure for microglial activation.

\section{Visual characterization}

Images were taken of the DGib, CA1 and PFC of the IBA- 1 stained sections at $\times 400$ magnification using quantitative imaging software (Leica QWin, Leica Microsystems). In each image, a circle was drawn which covered $0.06 \mathrm{~mm}^{2}$ of the original section. The microglia in this area were counted and morphologically characterized based on the activation stages 1-2 or 3-5 as described by Kreutzberg. ${ }^{[4]}$ Microglia characterized as being in stages 3-5 were considered to be activated. The number of activated 
microglia expressed as a percentage of the total number of microglia was used as a measure for microglial activation.

Quantifying morphological characteristics (cell size, cell body size, size dendritic processes and cell body size to cell size ratio)

As described in the previous section, images were taken of the DGib, CA1 and PFC of the IBA-1 stained sections. Several morphological characteristics of the stained microglia were analyzed by using image analysis software (Image-Pro Plus 6.0.0.26, Media Cybernetics, Inc. Rockville, USA). Figure 1 shows an overview of the method in microglia with a more quiescent morphology and microglia with a more activated morphology (for clarity purposes only a small section of the original picture are shown).

The area of interest was selected. The image analysis software automatically analyzes the picture and bases on the color intensity and distribution determines the background and intensity range of this area. The total cell size of all microglia in the area of interest was determined by counting all pixels that were darker than the background using the "automatic dark objects" function. The total cell body size was determined by applying an intensity threshold (histogram based manual intensity range selection) and size filter (area filter range) (similar to the method described by Tynan et al. ${ }^{[18]}$. With the intensity threshold only pixels with a staining intensity above a certain value in the intensity range scales, (0-255) are counted. Since IBA1 staining gives a higher staining intensity in the cell bodies than most of the dendritic processes applying an intensity threshold will filter out most dendritic processes from the analysis. In addition, the size filter excludes all pixel clusters that are smaller than a certain size from the analysis, filtering out any dendritic processes with a high-staining intensity. The intensity threshold and size filter depend on the overall intensity of the staining. In our case, the intensity threshold was 150-170 and the size filter was 100 pixels.

In addition, the number of cell bodies was counted to give the number of microglia in the area of interest. The total size of the dendritic processes was determined by subtracting the cell body size from the cell size. The total cell size, total cell body size and total size of dendritic processes were corrected for the number of microglia in the sample area to gain the average size, cell body size and size of dendritic processes for each microglial cell in the sample. Finally, the cell body to cell size ratio (\%) was determined and utilized as a measurement for microglial activation.

As an alternative to the analysis with Image-Pro (Image-Pro Plus 6.0.0.26, Media Cybernetics, Inc.), we performed a similar analysis by using ImageJ (ImageJ 1.48v, http://imagej.nih.gov/ij), a publically available image analysis package. The area of interest was selected. Using the adjusted threshold and analyze particles functions the intensity thresholds and size filter were applied. To measure the total cell size the threshold was maintained at the level that was automatically provided by the program, and no size filter was applied. To measure the total cell body size the threshold was lowered 40 points, and size filter of 150 pixels was applied.

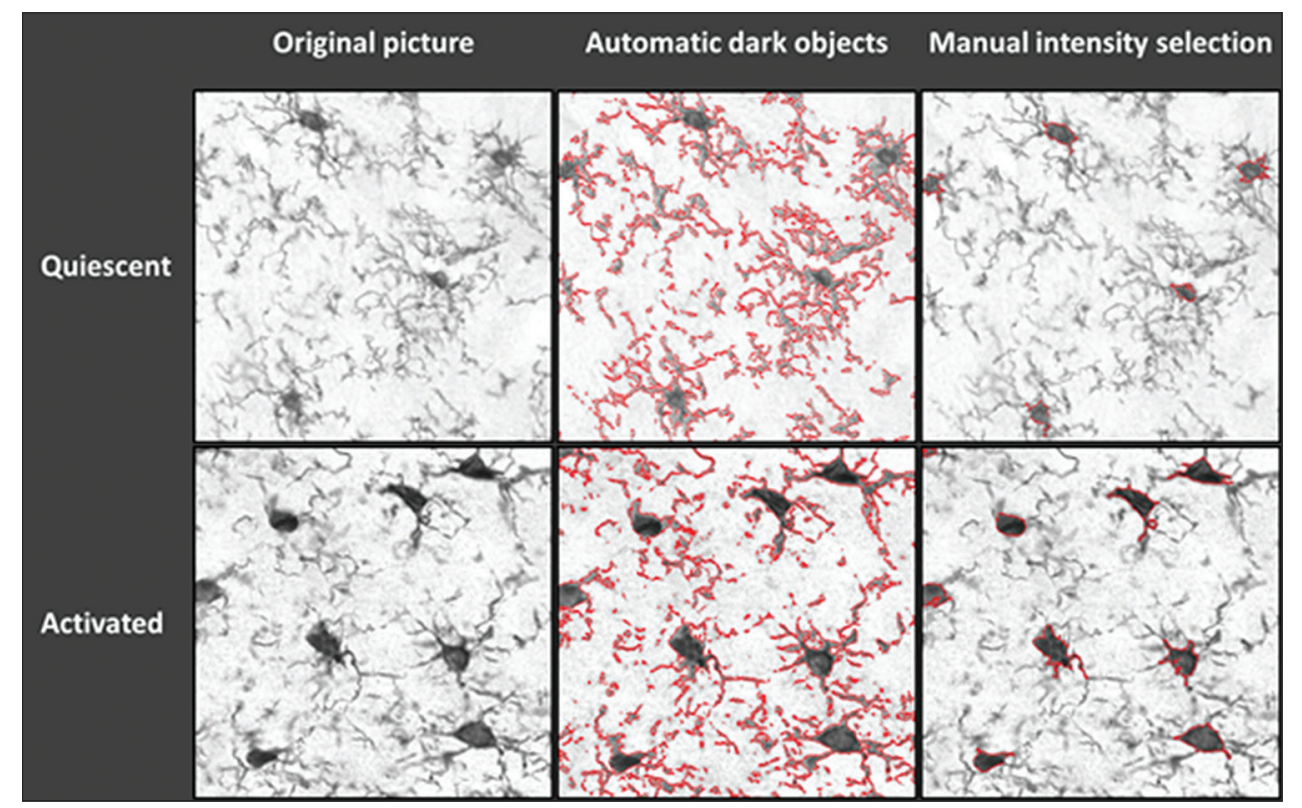

Figure 1: Illustration of the image analysis method used to quantify the morphological characteristics of microglia in ionized calcium-binding adaptor protein-1 stained sections (for clarity purposes only a small section of the original picture is shown). Left: the unprocessed pictures. Middle: all the pixels that are darker than the background are traced (red lines) to determine the total cell size of all microglia combined. Right: pixel-clusters that are above an applied staining threshold and size-filter are traced (red lines) to determine the total cell body size of all microglia combined, as well as the number of microglia 
Statistical analysis

All statistical analyses were performed using SPSS (IBM SPSS Statistics for Windows, 20.0.0.2, New York, USA). Means and confidence intervals are shown. Figures were prepared by using GraphPad Prism (version 5.00 for Windows, GraphPad Software, San Diego, California, USA). Pearson's correlation coefficients were determined to examine the relationship between the various measures for microglial activation for all individual brain areas, and all brain areas combined. The significance level for the Pearson's correlation coefficients was corrected by using Bonferonni method.

In addition, group comparisons were made for microglial activation in the CA1, DGib and PFC combined, using an analysis of variance with the experimental group as between subject factor followed by Tukey post-hoc analysis. Outcomes were considered to be significant when $P<0.05$.

\section{RESULTS}

Table 1 shows the average values of the outcome measures used to analyze microglia activation.

The image analysis by Image-Pro and ImageJ yielded highly comparable results as indicated by a strong correlation of the main outcome parameters cell body size $(r=0.792, P=0.000)$, size of dendritic processes ( $r=0.499, P=0.029)$ and cell body to cell size ratio $(r=0.918, P=0.000)$. Therefore, we have only used the outcomes of the analysis with Image-Pro for the rest of the study.

There is no significant correlation between OD and the other parameters used to measure microglia activation. The Pearson's correlations between the percentages of activated microglia based on visual characterization and the quantified morphological characteristics are shown in Table 2. In all separate brain area, the parameter of the cell body to cell size ratio is significantly and strongly correlated with microglial activation based on visual characterization. Therefore, this outcome may be a more accurate morphological characteristic to represent a marker of (classical) microglial activation.

Figure 2 shows a scatter plot of microglia activation based on morphological characterization and the cell body to cell size ratio for the three analyzed brain areas combined.

A comparison of the study outcome using the different analysis techniques is presented in Figure 3, which displays the average of densitometry measurements [Figure 3a], visual characterization [Figure 3b]

\section{Table I: Outcome measures microglial analysis}

\begin{tabular}{lcc} 
& $\boldsymbol{n}$ & Mean (95\% Cl) \\
\hline Activated microglia (stages 3-5) (\%) & 60 & $40.4(37.0-43.9)$ \\
Optical density & 60 & $0.17(0.16-0.18)$ \\
Number of microglia & 60 & $13.3(12.7-13.8)$ \\
Average cell size (pixels) & 59 & $2341(2227-2454)$ \\
Average cell body size (pixels) & 59 & $328(302-355)$ \\
Average size dendritic processes (pixels) & 59 & $2013(1910-2116)$ \\
Cell body to cell size ratio (\%) & 60 & $14.2(13.2-15.1)$ \\
\hline
\end{tabular}

Mean \pm SEM of the outcome measures used to analyze microglial activation. Average outcomes are representing an area of $0.06 \mathrm{~mm} \times 0.06 \mathrm{~mm}$ in the CA1, dentate gyrus inner blade and prefrontal cortex. The visual characterization outcomes and number of microglia have been previously published by Hovens et al. ${ }^{[1]} \mathrm{CA} 1$ : cornu ammonis $1 ; \mathrm{Cl}$ : confidence interval, SEM: standard error of the mean

\begin{tabular}{|c|c|c|c|c|c|}
\hline \multicolumn{6}{|c|}{$\begin{array}{l}\text { Table 2: Correlation between the percentages of } \\
\text { activated microglia based on visual characterization and } \\
\text { the quantified morphological characteristics of microglia }\end{array}$} \\
\hline & \multicolumn{5}{|c|}{ Morphological characteristics } \\
\hline & $\begin{array}{l}\text { Number } \\
\text { of cells }\end{array}$ & $\begin{array}{l}\text { Cell } \\
\text { size }\end{array}$ & $\begin{array}{l}\text { Cell } \\
\text { body } \\
\text { size }\end{array}$ & $\begin{array}{l}\text { Processes } \\
\text { size }\end{array}$ & $\begin{array}{l}\text { Cell } \\
\text { bodyl } \\
\text { cell }\end{array}$ \\
\hline \multicolumn{6}{|c|}{$\begin{array}{l}\text { Visual } \\
\text { characterization }\end{array}$} \\
\hline DGib & 0.137 & $0-0.449$ & $0.625^{\#}$ & $0-0.495$ & $0.839^{*}$ \\
\hline CA1 & 0.001 & $0-0.282$ & $0.796^{*}$ & $0-0.524$ & $0.890^{*}$ \\
\hline PFC & $0-0.390$ & $0-0.029$ & $0.680^{*}$ & $0-0.288$ & 0.944 * \\
\hline Total & $0-0.104$ & $0-0.170$ & $0.619^{*}$ & $0-0.343^{\#}$ & $0.855^{*}$ \\
\hline
\end{tabular}

Correlation (Pearson's R) between the percentage of activated microglia as determined by visual characterization according to Kreutzberg and the quantified morphological characteristics of microglia in the DGib $(n=20)$, hippocampal CA 1 region $(n=20), \operatorname{PFC}(n=20)$ and all three brain areas combined (total,

$n=60$ ). The morphological characteristics are: the number of microglia (number of cells), the average size of microglia (cell size), the average cell body size of microglia (cell body size), the average size of the dendritic processes of microglia (processes size) and the cell body to cell size ratio (cell body/cell) in the area of interest. ${ }^{\star} P<0.0013,{ }^{*} P=0.01$. PFC: prefrontal cortex; CA1: cornu ammonis 1; PFC: prefrontal cortex; DGib: dentate gyrus inner blade

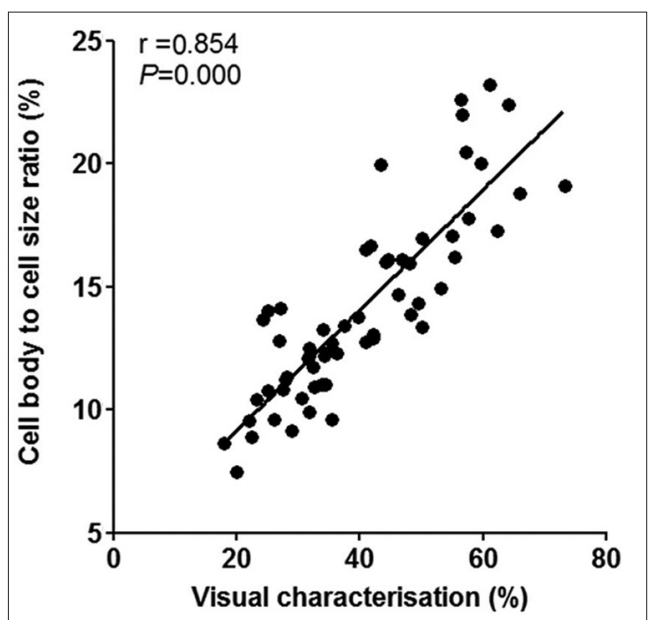

Figure 2: Relation of the cell body tot cell size ratio (cell body size as percentage of total cell size) with the percentage of activated microglia (activation stages 3-5) based on visual characterization for all rats in all analyzed brain areas. A regression line with associated Pearson's $r$ is shown

and our novel measurement, cell body to cell size ratio [Figure 3c]. Whereas the OD does not differ significantly between experimental groups, both the percentages of activated microglia based on visual characterization and the cell body to cell size ratio shows a significant difference between groups (visual 
characterization: $F_{3,56}=23.86, P=0.000$; cell body to cell size ration: $F_{3,56}=22.48, P=0.000$ ). Both of these measures for microglial activation show a similar pattern, with both groups of old rats showing increased microglial activation when compared to their young counterparts and further increase of microglial activation observed only in operated old rats.

Additional morphological characteristics of microglia in the IBA-1 stained sections are presented in Figure 4. Total cell size [Figure 4a] does not differ significantly among groups $\left(F_{3,56}=1.88, P=0.144\right)$. The total cell body size [Figure $4 \mathrm{~b}$ ] differs significantly among groups $\left(F_{3,56}=5.66, P=0.002\right)$, with a significant increase in cell body size only in old rats after surgery. The total size of the dendritic processes [Figure 4c] differs significantly among groups $\left(F_{3,56}=4.33\right.$, $P=0.008$ ) with a significant decrease in the size of dendritic processes only in old rats compared to their young counterparts; however no effect of surgery was observed.

The number of cell bodies in the areas of interest did not differ significantly in our experiment (young control: 14.0 [12.6-15.3]; young surgery: 13.9 [12.3-15.5]; old control: 12.5 [11.7-13.3]; old surgery: 12.8 [12.0-13.6]; $\left.F_{3,56}=0.89, P=0.454\right)$.

\section{DISCUSSION}

The aim of the current study was to validate a newly developed semi-automatic image analysis method to analyze microglial morphology as marker for microglia activation in IBA-1 stained brain sections of rat. For this purpose, we adapted a method previously developed by Vinet et al., ${ }^{[10]}$ using image analysis with an intensity threshold and size filter, to obtain the cell body size and the total cell size of microglia. We used these parameters as a measure for morphological changes and presented cell body

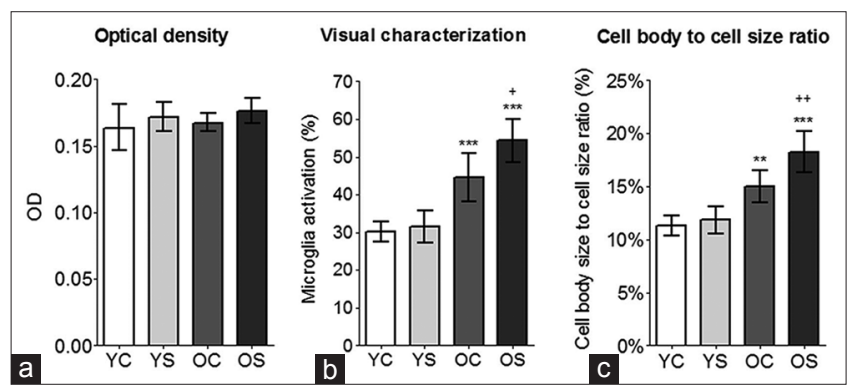

Figure 3: Potential markers for microglial activation in young and old rats with or without surgery. (a) The corrected optical density as marker for microglia activation; (b) The percentage of activated microglia (with an activation stage $\geq 3$ ) based on visual characterization. (c) The cell body to cell size ratio (\%) as marker for microglia activation. YC: young control; YS: young surgery; OC: old control; OS: old surgery. ${ }^{* \star} P<0.01$ and ${ }^{* \star *} P<0.001$ compared to young rats. ${ }^{+} P<0.05$ and ${ }^{++} P<0.01$ compared to age matched controls to cell size ratio as validated measure of microglial activation.

In this study, we compare different analysis methods for IBA-1 stained brain sections of young and aged rats with or without surgery. ${ }^{[17]}$ Surgery has been associated with the development of postoperative cognitive dysfunction (POCD), including impairment of memory, attention, and executive functions. ${ }^{[19-22]}$ Accumulating evidence indicates that surgery-induced (neuro) inflammation plays an important role in POCD development. ${ }^{[19,20,22-25]}$ Although patients of all ages can experience POCD, persisting and more severe problems are mainly seen in elderly surgical patients. ${ }^{[22,26]}$ Aging itself is also associated with neuroinflammatory changes in several brain regions, which may contribute to the increased incidence of POCD in elderly patients. ${ }^{[1,19,27]}$ Hence, our study design allows us to investigate whether different analysis methods can distinguish the effects of an intervention associated with pathophysiological changes, and the effects of aging, which could be considered to be a more physiological process.

The cell body to cell size ratio shows a very strong positive correlation with microglial activation determined by the visual characterization method. Both visual characterization and the newly developed method reveal similar alterations related to aging and surgery, validating the cell body to cell size ratio as a marker for (classical) microglial activation. However, no correlation was observed with densitometry, nor did densitometry measurements distinguish microglial changes related to aging and surgery could be obtained. Seemingly densitometry may have insufficient sensitivity to distinguish the relatively modest changes in IBA-1 protein expression that accompanies the microglial activity associated with aging and surgery. ${ }^{[19,20,23]}$ In addition, our new method provides outcome parameters that can be used for a more detailed analysis of microglia in an area of interest. Firstly, changes in the number of microglia can be determined. Second, by studying

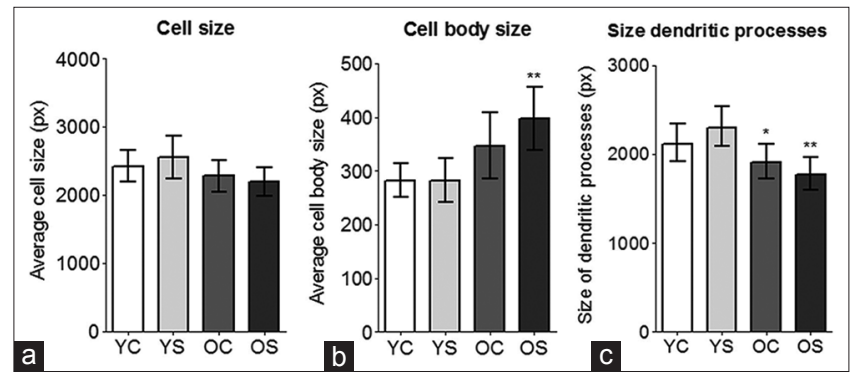

Figure 4: Morphological characteristics of microglia in young and old rats with or without surgery. (a) The total cell size (pixels) in the area of interest; (b) The total cell body size (pixels) in the area of interest; (c) The total size of the dendritic processes (pixels) in the area of interest. YC: young control; YS: young surgery; OC: old control; OS: old surgery. ${ }^{*} P<0.05$ and ${ }^{* \star} P<0.01$ compared to young rats 
the total microglial cell size, cell body size and size of the dendritic processes separately, the specific characteristics of the morphological changes can be investigated. In our experiment for instance, it became apparent that (without significantly changing the number of microglia or cell size) there is a reduction of the size of the dendritic processes. Surgery, on the other hand, led to increased cell body size, specifically in the aged rats. Accordingly, a dystrophic deramificated morphology of microglia has been observed in old rats and humans, ${ }^{[6,28]}$ whereas surgery was associated with a more classical microglial activation. ${ }^{[20,29]}$

The method proposed in this article has several limitations. Firstly, in this study we validated our method on relatively small areas $\left(0.06 \mathrm{~mm}^{2}\right)$ in order to compare it to microglial activation based on visual characterization in those same areas. Therefore, only approximately 13 microglia/area were analyzed. However, we successfully explored the method on substantially larger areas (data not shown), further confirming its benefits.

Second, although we aim for an objective marker for microglial activation, the staining threshold and size filter have to be subjectively determined by researchers based on the staining intensity of the dendritic processes and cell bodies. A possible solution is to standardize the value of the intensity threshold as compared to the intensity threshold of the background (provided by the program).

Third, the outcome of the analysis is largely dependent on the quality of the staining. Currently, we do not have a method automatically to filter out artifacts. To circumvent this problem, it is possible to analyze only those sections with good quality by manually indicating the area of interest. However, this makes the analysis more time consuming and may lead to bias.

We developed this method to analyze IBA-1 stained microglia in DAB stained sections. Theoretically the analysis could be applied to fluorescent staining. However, the analysis depends substantially on a clear visibility of the dendritic processes and with fluorescent staining this is not always the case. If dendrites are not clearly visible, only the cell body size could be measured as an alternative to our method.

Finally, in this study we only looked at age-related and surgery-induced microglial activation. Microglial activation in these cases is expected to be relatively subtle. Therefore, we currently cannot confirm whether our method is applicable for other conditions in which microglia activation occurs, such as the most extensive neuroinflammation in brain trauma.
In conclusion, the method for analyzing microglial morphological changes as marker for microglial activation by semi-automatic image analysis of IBA-1 stained brain sections, described in this article, provides a novel sensitive marker for microglial activation in rats, which is quick and easy to perform and provides additional information about the morphological characteristics of microglia. Although conclusions about microglial activation based on morphological characteristics alone should be drawn with great caution, this novel analysis method could, combined with other markers related to microglial activation, contribute to research in the field of neuroimmunology.

\section{ACKNOWLEDGMENTS}

We acknowledge funding from the University of Groningen as this research was part of the Rosalind Franklin Fellowship awarded to Dr. van Leeuwen. We thank Jan Keijser, Wanda Douwenga and Folkert Postema for their technical support and Kèvin Knoops for his assistance with implementing the image analysis method for Image J. We thank Prof. EA van der Zee, chair of the Department of Molecular neurobiology of the University of Groningen, and Prof. E Heineman, chair of the Department of Surgery of the University Medical Center Groningen for the use of their facilities and general and material support.

\section{REFERENCES}

1. Eggen BJ, Raj D, Hanisch UK, Boddeke HW. Microglial phenotype and adaptation. J Neuroimmune Pharmacol 2013;8:807-23.

2. Ransohoff RM, Perry VH. Microglial physiology: unique stimuli, specialized responses. Annu Rev Immunol 2009;27:119-45.

3. Nimmerjahn A, Kirchhoff F, Helmchen F. Resting microglial cells are highly dynamic surveillants of brain parenchyma in vivo. Science 2005;308:1314-8.

4. Kreutzberg GW. Microglia: a sensor for pathological events in the CNS. Trends Neurosci 1996;19:312-8.

5. Kettenmann H, Hanisch UK, Noda M, Verkhratsky A. Physiology of microglia. Physiol Rev 2011;91:461-553.

6. Olah M, Biber K, Vinet J, Boddeke HW. Microglia phenotype diversity. CNS Neurol Disord Drug Targets 2011;10:108-18.

7. Kaushik DK, Basu A. A friend in need may not be a friend indeed: role of microglia in neurodegenerative diseases. CNS Neurol Disord Drug Targets 2013;12:726-40.

8. Imai Y, Ibata I, Ito D, Ohsawa K, Kohsaka S. A novel gene iba1 in the major histocompatibility complex class III region encoding an EF hand protein expressed in a monocytic lineage. Biochem Biophys Res Commun 1996;224:855-62.

9. Ahmed Z, Shaw G, Sharma VP, Yang C, McGowan E, Dickson DW Actin-binding proteins coronin-1a and IBA-1 are effective microglial markers for immunohistochemistry. $J$ Histochem Cytochem 2007;55:687-700.

10. Vinet J, Weering HR, Heinrich A, Kälin RE, Wegner A, Brouwer N, Heppner FL, Rooijen Nv, Boddeke HW, Biber K. Neuroprotective function for ramified microglia in hippocampal excitotoxicity. J Neuroinflammation 2012;9:27.

11. Sasaki Y, Ohsawa K, Kanazawa H, Kohsaka S, Imai Y. Iba1 is an actin-cross-linking protein in macrophages/microglia. Biochem Biophys Res Commun 2001;286:292-7.

12. Hinwood M, Morandini J, Day TA, Walker FR. Evidence that microglia mediate the neurobiological effects of chronic 
psychological stress on the medial prefrontal cortex. Cereb Cortex 2012;22:1442-54.

13. Morrison HW, Filosa JA. A quantitative spatiotemporal analysis of microglia morphology during ischemic stroke and reperfusion. J Neuroinflammation 2013;10:4.

14. Karperien A, Ahammer H, Jelinek HF. Quantitating the subtleties of microglial morphology with fractal analysis. Front Cell Neurosci 2013;7:3.

15. Hinwood M, Tynan RJ, Charnley JL, Beynon SB, Day TA, Walker FR. Chronic stress induced remodeling of the prefrontal cortex: structural re-organization of microglia and the inhibitory effect of minocycline. Cereb Cortex 2013;23:1784-97.

16. Franciosi S, Ryu JK, Shim Y, Hill A, Connolly C, Hayden MR, McLarnon JG, Leavitt BR. Age-dependent neurovascular abnormalities and altered microglial morphology in the YAC128 mouse model of Huntington disease. Neurobiol Dis 2012;45:438-49.

17. Hovens IB, Schoemaker RG, van der Zee EA, Heineman E, Nyakas C, van Leeuwen BL. Surgery-induced behavioral changes in aged rats. Exp Gerontol 2013;48:1204-11.

18. Tynan RJ, Naicker S, Hinwood M, Nalivaiko E, Buller KM, Pow DV, Day TA, Walker FR. Chronic stress alters the density and morphology of microglia in a subset of stress-responsive brain regions. Brain Behav Immun 2010;24:1058-68.

19. Barrientos RM, Hein AM, Frank MG, Watkins LR, Maier SF. Intracisternal interleukin-1 receptor antagonist prevents postoperative cognitive decline and neuroinflammatory response in aged rats. J Neurosci 2012;32:14641-8.

20. Cibelli M, Fidalgo AR, Terrando N, Ma D, Monaco C, Feldmann M, Takata M, Lever IJ, Nanchahal J, Fanselow MS, Maze M. Role of interleukin-1beta in postoperative cognitive dysfunction. Ann Neurol 2010;68:360-8.

21. Price CC, Garvan CW, Monk TG. Type and severity of cognitive decline in older adults after noncardiac surgery. Anesthesiology 2008;108:8-17.

22. Hovens IB, Schoemaker RG, van der Zee EA, Heineman E, Izaks GJ, van Leeuwen BL. Thinking through postoperative cognitive dysfunction: how to bridge the gap between clinical and pre-clinical perspectives. Brain Behav Immun 2012;26:1169-79.

23. Hovens IB, Schoemaker RG, van der Zee EA, Absalom AR, Heineman E, van Leeuwen BL. Postoperative cognitive dysfunction: involvement of neuroinflammation and neuronal functioning. Brain Behav Immun 2014;38:202-10.

24. Price CC, Tanner JJ, Schmalfuss I, Garvan CW, Gearen P, Dickey D, Heilman K, McDonagh DL, Libon DJ, Leonard C, Bowers D, Monk TG. A pilot study evaluating presurgery neuroanatomical biomarkers for postoperative cognitive decline after total knee arthroplasty in older adults. Anesthesiology 2014;120:601-13.

25. Peng L, Xu L, Ouyang W. Role of peripheral inflammatory markers in postoperative cognitive dysfunction (POCD): a meta-analysis. PLoS One 2013;8:e79624.

26. Monk TG, Weldon BC, Garvan CW, Dede DE, van der Aa MT, Heilman KM, Gravenstein JS. Predictors of cognitive dysfunction after major noncardiac surgery. Anesthesiology 2008;108:18-30.

27. Dilger RN, Johnson RW. Aging, microglial cell priming, and the discordant central inflammatory response to signals from the peripheral immune system. $J$ Leukoc Biol 2008;84:932-9.

28. von Bernhardi R, Tichauer JE, Eugenín J. Aging-dependent changes of microglial cells and their relevance for neurodegenerative disorders. J Neurochem 2010;112:1099-114.

29. Wan Y, Xu J, Ma D, Zeng Y, Cibelli M, Maze M. Postoperative impairment of cognitive function in rats: a possible role for cytokine-mediated inflammation in the hippocampus. Anesthesiology 2007;106:436-43.

Cite this article as: Hovens IB, Nyakas C, Schoemaker RG. A novel method for evaluating microglial activation using ionized calcium-binding adaptor protein-1 staining: cell body to cell size ratio. Neuroimmunol Neuroinflammation 2014;1(2):82-8.

Source of Support: Nil. Conflict of Interest: No.

Received: 23-05-2014; Accepted: 18-07-2014 